\title{
Sunflower Seed Cake and Larvae MassRheological Properties Analysis During Pressing WithVarying Temperature, Pressure and Oil Content
}

\section{Ivan Shorstkii, Alexandr Gukasyan, Evgeny Koshevoi, and Vyacheslav Kosachev}

Department of Technological Equipment and Life-Support Systems, Kuban State University of Technology, 2 Moskovskayast., Krasnodar 350072, Russian Federation

Abstract. For the successful implementation of alternative protein sourcesforbiorefinery, optimization of the process parameters is crucial. Knowledge of the rheological propertiesis necessary for the design and development of appropriate equipment and process calculations. The objective of this research was to evaluate the effect of the following pre-treatments: temperature, pressure andeffect of initial oil content on the rheological properties of sunflower seedcake and larvae tissue. The rheological behavior of two protein sourceswas determined by using a rotational viscometer

Corresponding Author: Ivan Shorstkii; email:

i-shorstky@mail.ru

\section{Dates}

Published 13 January 2022

Publishing services provided by Knowledge E

(c) Ivan Shorstkii et al. This article is distributed under the terms of the

Attribution License, which permits unrestricted use and redistribution provided that the original author and source are credited.

Selection and Peer-review under the responsibility of the 8th Scientific and Practical Conference Conference Committee.

\section{G OPEN ACCESS} with a hydraulic system and thermostatic bath attached to the equipment. Using the mathematical apparatus and experimental data it was observed that the plastic viscosity of the sunflower seed cake corresponded to the viscosity of the vegetable oil, which confirmed the Bingham rheology assumption put forward in this work. For the larvae mass, a Hershey Buckley fluid model was proposed.A positive linear relationship was found for pressure and a negativelinear relationship was found for the oil content of the sunflower seed cake and larvae tissue on shear stress.

Keywords: rheological property, sunflower seed cake, larvae, pulsed electricaldischarge, viscoplasticity flow, Bingham model, modelling

\section{Introduction}

Sunflower oil production is rapidly emerging in Russia, Ukraine, Turkey and other countries, filling an important niche of locally supplied protein and fat sources. Processing of sunflower seeds is becoming of outmost importance to fulfill the requirements for safe products and find efficient ways to reduce potential chemical and technological hazards [1].

Due to their potential dietary contribution regarded to be suitable for animals feeding and human consumption, and at the same time as a sustainable, along with isolation of relevant nutritional ingredients such as protein or fat, the number of insect varieties and 
farms increases steadily [2]. Insect biomass contains from 40 to $70 \%$ of crude protein and up to $36 \%$ of lipids that can be extracted and used for various purposes [3].

Studying rheological properties of the samples pre-treatedbytemperature, ovepressure and initial oil contentcan help to advance work and to develop projects for their industrial application. Such technology has great prospect in food and pharmaceutical industry, because it can increase the yield of extra virginvegetable oil during pressing. Some studies of the influence of differentpre-treatment on rheological properties on some materials have been reported in scientific literature [4-6].

Some studies of the influence of particle size and temperature on sunflower paste have also been reported [7-9]; however, there is a gap regarding the influence of overpressure and initial oil content on viscosity in the initial stages of oils processing.

The purpose of this paper is to study rheological parameters peculiar for the evaluation of influence oftemperature, hydrostatic pressure and material oil content for sunflower seed cake and larvae mass. Rheology study of five different protocols with parameters close to the conditions of the production cycle were carried out.

\section{Materials and methods}

\subsection{Preparation of seed cake}

Sunflower seed cakewas obtained from a local factory afterindustriallyflaking and cooking steps before pressing operation. Such industrial procedures allowed us to obtain sunflower mass close to the homogeneous structure. Initial oil content of seed cake according to the specification was $56.1 \pm 0.3 \%$. Initial moisture and ash content were $5.0 \pm 0.7 \%$ and $10.2 \pm 0.6 \%$ respectively.Protein content of seed cake according to the specification was $16.2 \pm 0.8 \%$.

\section{Preparation of larvae mass}

For the experiments live black soldier fly larvae (Hermetiaillucens) were purchased from a local German producer (Ahaus, Germany). The larvae were stored for 24 hours at $4{ }^{\circ} \mathrm{C}$ until the experiments. Prior to the trials, the samples were sieved to remove residual faeces and milled using shredder until a homogeneous mass was obtained. Initial oil content of larvae mass was $34.5 \pm 1.3 \%$. Initial moisture content was72.0 \pm 1.7\%. Protein content of larvae mass according to the specification was $60.8 \pm 0.7 \%$. 


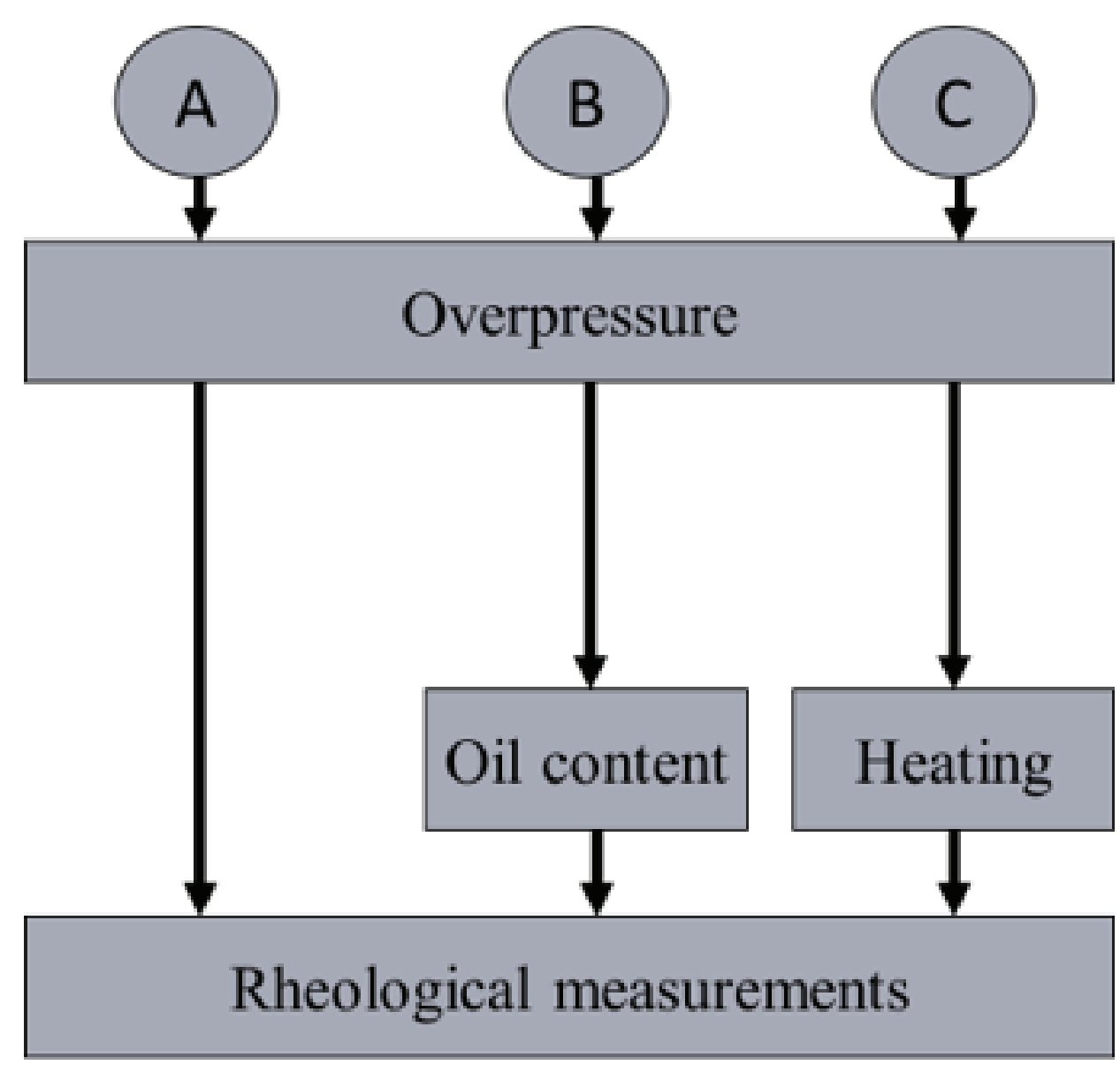

Figure 1: Different protocols of rheological measurements.

\subsection{Rheological measurements}

\subsubsection{Measurement protocols}

Rheological measurement was produced according to the scheme shown in Figure 1. Different protocols of rheological measurement involving heating, overpressure and different oil content were compared:

1. Protocol A: overpressure(in range from 980 up to $2800 \mathrm{~Pa}$ ) + rheological measurements;

2. Protocol B: overpressure (at $981 \mathrm{~Pa}$ for sunflower seed cake and $2700 \mathrm{~Pa}$ for larvae mass) + variation of oil content (from $40 \%$ up to $56 \%$ for sunflower seed cake and from $25 \%$ up to $34.5 \%$ for larvae mass) + rheological measurements;

3. Protocol C: overpressure (at $1805 \mathrm{~Pa}$ ) + heating(in range from 301 up to $318 \mathrm{~K}$ ) + rheological measurements; 


\subsubsection{Rheological measurements}

The rheological behavior of sunflower seed cake was determined by using a rotational viscometer Fungilab One Pro (Fungilab, Spain) with cylindrical spindle L4. Rheological analyses were obtained with shear rate from 1 to $10-1$.

\subsubsection{Rheological measurements with adjusting temperature, over- pressure and oil content}

The choice of a rotational measurement method is based on the ability to measure the viscosity of both Newtonian and non-Newtonian media [10]. To assess the effect of pressure, the viscometer cell was equipped with a hydraulic system of controlled overpressure on the viscometer rotor [11]. Under the influence of gravity, the piston pressed on the mass under study. The value of excess pressure was determined as overpressure $=\mathrm{Fg} / \mathrm{S}$, where $\mathrm{Fg}$ - gravity, $\mathrm{H} ; \mathrm{S}$ - area of the piston, $\mathrm{m}^{2}$. The gap between the rotor and the piston was $2 \mathrm{~mm}$. To minimize the measurement error with and without the hydraulic system, the removal of the effective viscosity indicators from the device was performed with three times repetition. The reliability of experimental data was evaluated by analyzing the variance index and the average absolute deviation. To change the temperature, the viscometer cell was equipped with a flexible coil, which was supplied with hot water from the thermostat. The cell along with the coil was located in a foam body to ensure uniform heating of the pulp by volume. To change the oil content, the pulp was subjected to preliminary mechanical pressing on a hydraulic press, thoroughly mixed and sent to the measured cell of the viscometer. Thus, the experimental study of the effective viscosity of the pulp was carried out with a variation in the values of overpressure in the range from 980 to $2,700 \mathrm{~Pa}$, temperature in the range from 28 to $45^{\circ} \mathrm{C}$ and oil content of sunflower seed cake at values of $40,48.5$ and $56 \%$.The values used to fitting the data to the model related to the downward curve of the shear rate. The model adjustment was performed by using the Microsoft Excel software.

\subsection{Microstructure}

Sunflowerseed cake samples were examined using a scanning electron microscope (SEM) JEOL SEM 6360LA (A*Star, IMRE, Singapore) at the accelerating voltage of $10 \mathrm{kV}$ and at the medium magnification of $170 x$. 
Whole $\mathrm{H}$. illucens larvae were fixed in a SEM sample holder with special optimal cutting temperature compound (OCT compound) glue consisting of glycols and resins, cryogenic frozen in super-cooled liquid nitrogen and inserted in a cryogenic preparation system (Emitech K 1250, UK), where the samples were broken down, and the free water was removed by sublimation. The prepared samples were transferred into the SEM (Jeol JSM 6460 LV, Japan) at approximately $-180{ }^{\circ} \mathrm{C}$ and analyzed.

\subsection{Statistical analysis}

To minimize the magnitude of the measurement error with and without a hydraulic system, measurements of the apparent viscosity from the device was performed with triplicate. The results expressed as means \pm standard deviation. Statistical significance was declared at $p<0.05$ tested by analysis of variance (ANOVA).All statistical analyses including ANOVA were calculated using IBM SPSS Statistics Subscription software.

\section{Results and discussion}

\subsection{Sunflower seed cake microstructure}

The structure of rheological flows in the oil press auger is largely determined by the choice of the rheological flow equation, which affects the volumetric capacity of the oil press. When analyzing the microphotography of the pulp, oil globules $(\mathrm{O})$ in the dark and light zones are clearly visible on its surface, as well as the oil film (F), expressed as light homogeneous areas (Fig. 2). The membrane of the oilseed cells (M) is represented as ringed light fibers surrounding the oilseed cell. At rest, the pulp is a loose coagulation structure [12]. Adhesion between dispersed particles occurs mainly due to free oil released as a result of heat treatment. In this way, the contacting particles of the pulp form a frame in a stationary oily film, linked to the walls of the screw channel.

The cross section cut of larvae sample is shown on Figure 2 . The insect intestines can be observed from Figure 2. When analyzing SEM-images of larvae tissue, oil globules $(\mathrm{O})$ are clearly visible on the surface of the sample. The size of oil globules ranges from a few microns up to $40 \mu \mathrm{m}$.

\subsection{Flow characteristics}




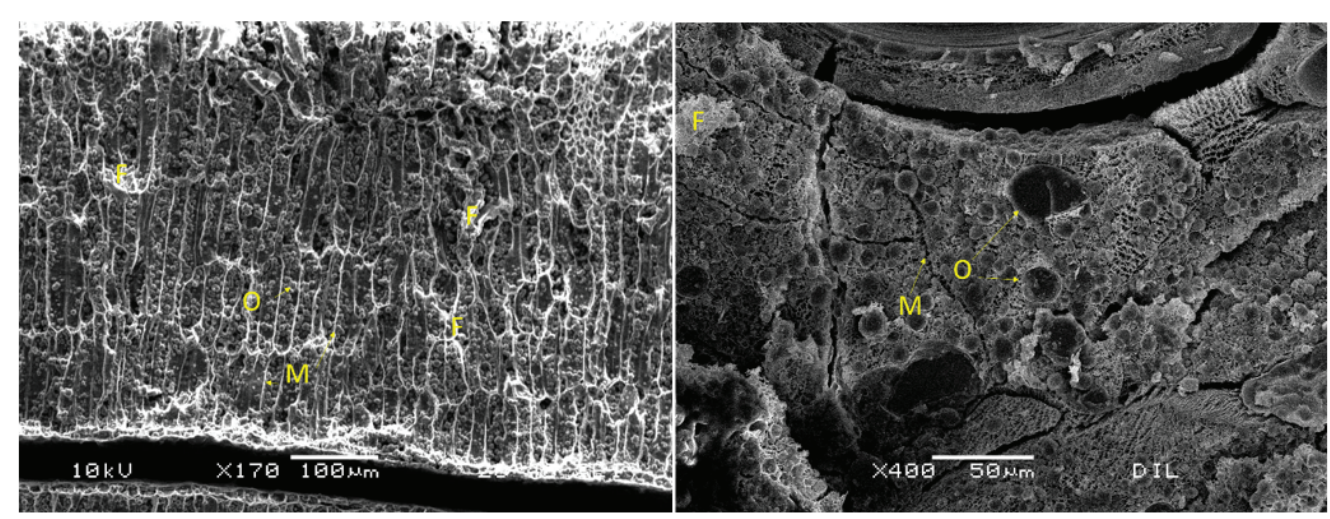

Figure 2: SEM-image of sunflower tissue (left) and larvae tissue (right) at x170 and $\times 400$ magnification.
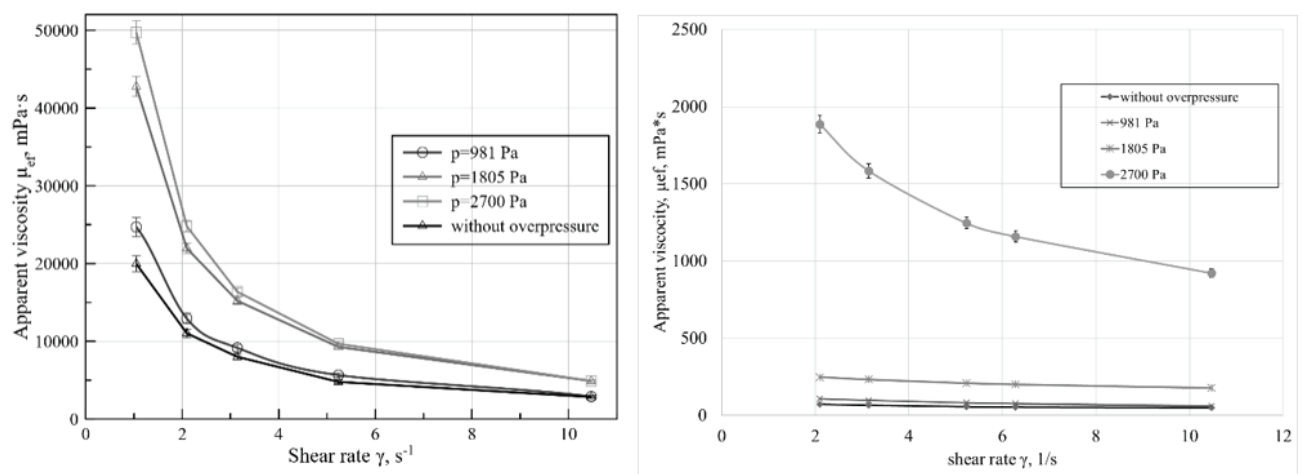

Figure 3: Apparent viscosity and shear rate dependence of seed cake (left) and larvae mass (right) at 981, 1805 and 2700 Pa over-pressure.

\subsubsection{Effect of PEDtreatment (protocol A)}

Structurally seed cake is a complicated dispersed system consisting of a disperse phase bubbles, oil globules with husk droplets and disperse medium as a protein shell.

The steady-shear rate flow of sunflower seed cake with different overpressure value was evaluated. Figure 3 shows the viscosity for protocol A sunflower seed as a function of the shear rate. Non-Newtonian shear-thinning behavior from the flow curves sunflower seed cake (pre-treated and non-treated) exhibit is observed. A similar shearthinning behavior has also been observed for sesame seed [13] and sage seed solutions [14].

Because oilseed material had inhomogeneous loose structure in comparison with cellular structure of fruits and vegetables, only a few layers of surface oil cells were damaged and oil droplets released on the surface. Flow curve $\mu_{e f}(\gamma)$ showed that apparent viscosity decreased with decreased value ofoverpressure $(981,1805$ and 2700 $\mathrm{Pa})$. 
TABLE 1: Rheological model parametersfor non-treatedsunflowerseed cake

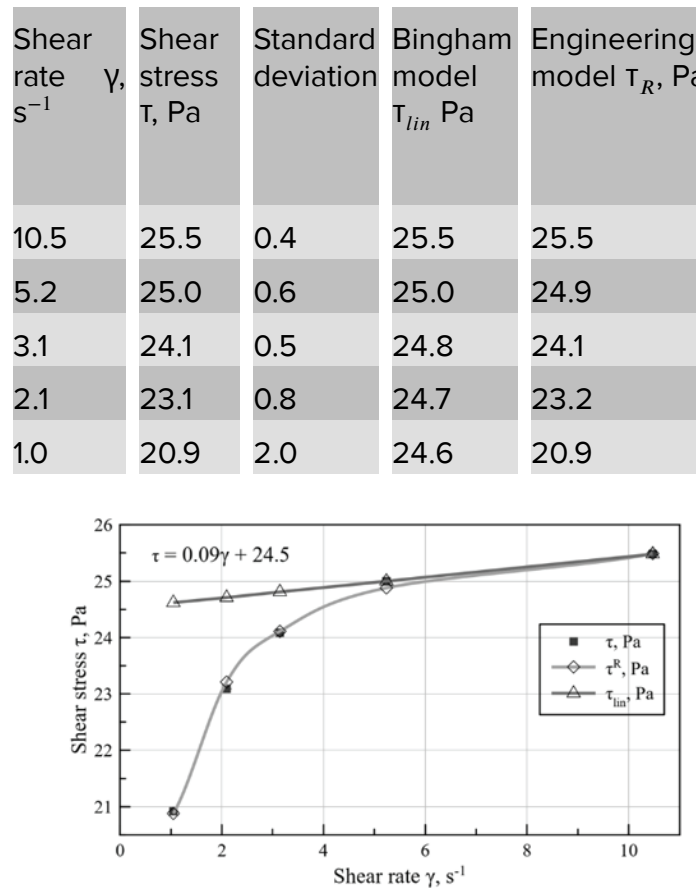

\begin{tabular}{|c|c|c|}
\hline $\begin{array}{l}\text { Discrepancy } \\
\text { of Bingham } \\
\text { model (T- } \\
\left.\mathrm{T}_{\text {lin }}\right) / \mathrm{T}\end{array}$ & $\begin{array}{l}\text { Discrepancy } \\
\text { of } \\
\text { engineering } \\
\text { model } \\
\left.\mathrm{T}_{R}\right) / \mathrm{T}-\end{array}$ & $\begin{array}{l}\text { Confidence } \\
\text { interval } \delta_{\tau} / \top\end{array}$ \\
\hline $0.0 \%$ & $0.0 \%$ & $1.8 \%$ \\
\hline $0.0 \%$ & $0.5 \%$ & $2.4 \%$ \\
\hline $3.0 \%$ & $0.1 \%$ & $2.2 \%$ \\
\hline $7.0 \%$ & $0.5 \%$ & $3.3 \%$ \\
\hline $17.7 \%$ & $0.2 \%$ & $9.6 \%$ \\
\hline
\end{tabular}

Figure 4: Shear stress vs. shear rate diagram for sunflower seed cake (left) and larvae mass (right).

Binghamand engineering model for oil presses were fitted to experimental shearrate/shear-stress data fornon-treated sunflower seed cake (table 1). A linearization of initial rheological parameters in the inverse value of $1 / \gamma$ and $1 / T$ was used to approximate data in Table 1.The engineering rheological model for sunflower seed cake in oil press can be represented as [15]:

$$
\tau^{R}(\dot{\gamma})=\frac{1}{b_{0}+\frac{b_{1}}{\dot{\gamma}}}
$$

Here $b_{0}, b_{1}$ are linear approximation coefficients of the reciprocals flow parameters of the material in the oil press channel $\left(\mathrm{b}_{0}=0.04 \mathrm{~Pa}^{-1}\right)$ and $\left(\mathrm{b}_{1}=0.01 \mathrm{~Hz} / \mathrm{Pa}\right)$.

Considering that apparent viscosity showed nearly Newtonian character at higher shear rates to determine the exact relationship between viscosities and shear rates, shear stress vs. shear rate dependence of sunflower seed cake was plotted (Figure 4). At low shear rate $\left(\gamma<5 \mathrm{~s}^{-1}\right)$ the external particles of sunflower seed cake slide along the channel walls, and with an increase in the shear rate on the oil film.

For larvae mass the flow curves ofshear rate and stress demonstrates sheardiminishing conduct $(n<1)$ with a yield value (Figure 4).The yield stress characterized as slightest shearstress important to start product flow, linkedwith the breaking of the material's innerstructure. 
TABLE 2: Values for the parameters of the Herschel-Buckley model for the larvae mass

\begin{tabular}{|l|l|l|l|l|}
\hline Temperature, $\mathrm{K}$ & $\mathrm{T}_{0}, \mathrm{~Pa}$ & $\mathrm{~K},\left(\mathrm{~Pa}^{*} \mathrm{~s}^{n}\right)$ & $\mathrm{n}$ & $\mathrm{R}^{2}$ \\
\hline 301 & 1.03 & 2.35 & 0.43 & 0.979 \\
\hline
\end{tabular}

Herschel-Buckley's (HB) modelcan be represented as [16]:

$$
\mathrm{T}=\mathrm{T}_{0}+K \bullet \mathrm{Y}^{n}
$$

where $\mathrm{K}$ - the consistency index; $\mathrm{n}$ - the flow index.

Elastic deformation happens underneath the yield stress making it act like an elastic solid; however material flows over the yield stress making it act like a viscous liquid. If there should arise an occurrence of multiphase material like vegetable puree/paste, which is formed by a dispersion of insoluble parts (materials of cell wall) in a water solution (serum, containing sugars, minerals, proteins, and solvent polysaccharides), is having a yield stress [16]. Table 2, describes the estimations of the Herschel-Buckleymodel parameters for larvae mass. A higher estimation of $\mathrm{R}^{2}$ (more than 0.97 ) was obtained. A nearby value for yield stress, flow index ( $\mathrm{n})$, and consistency index $(K)$ were acquired tentatively when contrasted with values from the literature for vegetable items.

The rheological engineering model of sunflowerseed cake flow represented by eq.(1)allows to determine the infinite stress value $\mathrm{T}_{\infty}$ as asymptote to stress curve, determined by the following equation:

$$
\tau_{\infty}=\lim _{\dot{\gamma} \rightarrow \infty}\left[\tau^{R}(\dot{\gamma})\right]=\frac{1}{b_{0}}
$$

To clarify the parameters of rheological engineering model (1)a smooth functional relationship in the form of spline approximation at the points of shear stress vs. shear rate diagram (Figure 4 ) on the interval $[a=1.0 ; b=10.5] \mathrm{Hz}$ is required (Table 1). Cubic spline used to approximate flow curve. Cubic spline represents benefits ofa function that:

on each segment is a polynomial of degree higher than three;

has continuous first and second derivatives on the whole interval [a, b];

in the experimental points, the equality of the spline interpolation function is done. For the unambiguous assignment of the spline impose additional requirements on the borders of the spline:T"(a) = T" $(b)=0$. In this case, according to Schoenberg-Whitney about the conditions of existence of a spline interpolation there is only one spline $\mathrm{T}_{s}(\gamma)$ 
satisfying the above conditions. In this case the relative discrepancy of rheological engineering model can be represented by the objective function $Z\left(b_{0}, b_{1}\right)$ :

$$
Z\left(b_{0}, b_{1}\right)=\int_{a}^{b}\left[\frac{\tau^{R}(\gamma)-\tau_{s}(\gamma)}{\tau^{R}(\gamma)}\right]^{2} d \gamma
$$

Minimization of the functional (3) allowed us to refine the parameters of the engineering model in comparison with their quasi-linear approximation $\left(\mathrm{bO}=0.0356 \mathrm{~Pa}^{-1}\right.$ ) and $(\mathrm{b} 1=0.0043 \mathrm{~Hz} / \mathrm{Pa}$ ). The realistic flow equation that is typical for an oil press in the process of extracting oil from the pulp is determined by the interval of shear rates: from 5 to $11 \mathrm{rad} / \mathrm{sec}$. In this case, to determine the rheological parameters of the sunflower seed cake flow in the auger channel, the most realistic flow equation is the ideal-plastic Bingham model:

$$
\tau(\dot{\gamma})=\tau_{0}+\mu_{p l} \cdot \dot{\gamma}
$$

where $\mathrm{T}_{0}$ - Bingham yield stress; $\mu_{p l}$ - plastic viscosity. The parameters of the equation(5) can be determined on the basis of a linear approximation in the specified range of shear rates found with the asymptotes from eq.(3) of the parameters of engineering models (1). From the graphs of linear approximations (Figure 4) parameters $\mathrm{T}_{0}=24.5$ $\mathrm{Pa}$ and $\mu_{p l}=0.09 \mathrm{~Pa} \cdot \mathrm{s}$ for non-treated seed cake and $\mathrm{T}_{0}=22.9 \mathrm{~Pa} ; \mu_{p l}=0.11 \mathrm{~Pa} \bullet \mathrm{s}$ for PED-treated seed cakewere found. From an initial approximation of $\mathrm{T}_{0}$ and $\mu_{p l}$ for shear rates in range from 5.2 to 10.5 by relative discrepancy of ideal Bingham plastic model(5) can beobtained:

$$
Z_{B}\left(\tau_{0}, \mu_{p l}\right)=\int_{5.2}^{10.5}\left[\frac{\tau^{R}(\dot{\gamma})-\tau(\dot{\gamma})}{\tau^{R}(\dot{\gamma})}\right]^{2} d \dot{\gamma}
$$

Minimization of $Z_{B}\left(T_{0}, \mu_{p l}\right)$ from equations (6) allowed to specify the parameters of the ideal Bingham-plastic model with respect to engineering rheological functions $\left(\square_{0}\right.$ $\left.=24.4 \mathrm{~Pa} ; \mu_{p l}=0.12 \mathrm{~Pa} \bullet \mathrm{s}\right)$ for non-treated sunflower seed cake and $\left(\square_{0}=22.7 \mathrm{~Pa}\right.$; $\mu_{p l}=0.11$ Pa $\bullet$ ) for PED-treated sunflower seed cake. Considering the fact that the apparent viscosity of sunflower seed cake corresponds to the viscosity of vegetable oil the Bingham model for sunflower seed cake was confirmed. 

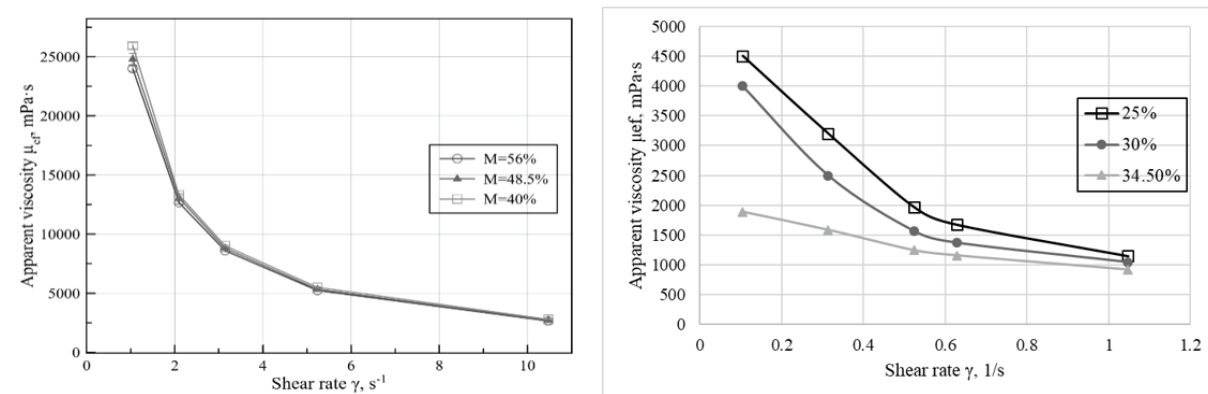

Figure 5: Apparent viscosity and shear rate dependence of sunflower seed cake at $56,48,5$ and $40 \%$ oil content at $\mathrm{p}=981 \mathrm{~Pa}$ (left) and larvae mass at $34.5,30$ and $25 \%$ oil content at $\mathrm{p}=2700 \mathrm{~Pa}$ (right).

TABLE 3

\begin{tabular}{l|l|l} 
Oil content, M / 100 & Yield stress, $\mathrm{T}_{0}$ & Plastic viscosity, $\mu_{p l}$ \\
$40 \%$ & $28.5 \mathrm{~Pa}$ & $42.8 \mathrm{mPa} \cdot \mathrm{s}$ \\
$48.5 \%$ & $27.5 \mathrm{~Pa}$ & $54.1 \mathrm{mPa} \cdot \mathrm{s}$ \\
$56 \%$ & $27.1 \mathrm{~Pa}$ & $60.3 \mathrm{mPa} \cdot \mathrm{s}$
\end{tabular}

\subsection{Effect of oil content (Protocol B)}

In Fig. 5 the viscosity diagrams ( $\mathrm{mPa} \cdot \mathrm{s})$ vs. shear rate $\left(\mathrm{s}^{-1}\right)$ are represented for seed cake measured at oil content of $40,48,5$ and $56 \%$ at overpressure $\mathrm{p}=981 \mathrm{~Pa}$ and for larvae mass at oil content of 25, 30 and $34.5 \%$ at overpressure $p=2700 \mathrm{~Pa}$.

To assess the influence of the oil content of sunflower seed cake on the parameters of the Bingham rheological model, a statistical analysis of the coefficients of linear approximation of experimental data was performed (Fig. 5), which showed the dependence only of the yield strength and effective viscosity on the oil content of the pulp. Reducing the oil content leads to an increase in the expended pressing energy and, as a result, an increase in the temperature of the pulp and the pressure on the matrix of the oil press. The presence of free oils in the form of a film on the surface of the pulp provides a powerful lubricating effect [17]. In addition, the presence of even $1 \%$ oil on the surface of the pulp provides stabilization and normalization of the pressing process, which indicates the importance of the process of heat treatment. The parameters of equation (4) are determined from the graphs of linear approximations for the oilseeds $m=40,48.5$ and $56 \%$, which are listed in table. 2 . at the same time, the plastic viscosity values that characterize the presence of an oil film are in good agreement with the viscosity values of sunflower oil [18].

Table 3

Bingham equation parameters for different oil content in seed cake

Oil content effect on parameters of equation (4) can be represented as: 

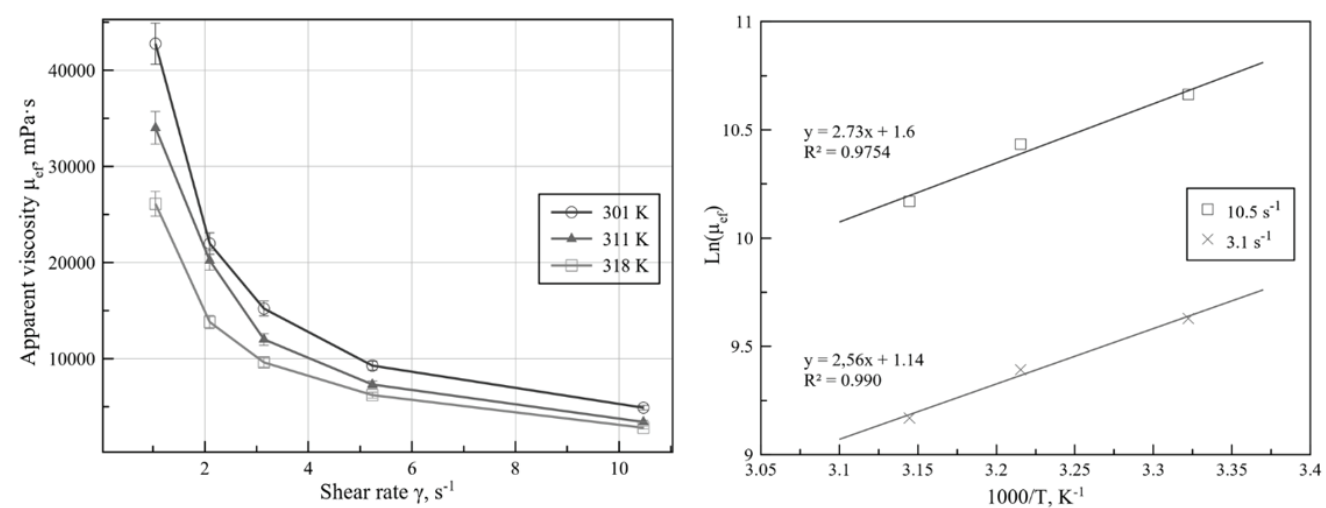

Figure 6: Apparent viscosity and shear rate dependence of sunflower seed cake at 301, 311 and $318 \mathrm{~K}$ (left) and temperature ramp dependence on natural logarithm at $p=1805 \mathrm{~Pa}$.

$$
\tau(\dot{\gamma}, M)=(-0.09 \cdot M+31.9 P a)+(0.0011 \cdot M-0.0005 \cdot P a \cdot s) \cdot \dot{\gamma}
$$

\subsubsection{Effectof temperature(Protocol C)}

Of practical interest is the study of the influence of temperature on the rheological model of the flow of sunflower pulp. During the pressing process, the temperature of the pulp can reach $140{ }^{\circ} \mathrm{C}$. The change of rheological properties of pulp at different temperatures is due to the spatial-temperature structural changes in the volume of pulp, as well as the dynamic process of structure formation. In this regard, the preheating process of the pulp is an important preparatory stage that affects the efficiency of the pressing process.In fig. 6 the viscosity diagrams (mPa.s) vs. shear rate $\left(\mathrm{s}^{-1}\right)$ are represented for seed cake measured at temperature of 301,311 and $318 \mathrm{~K}$. All samples from protocol C were under $1805 \mathrm{~Pa}$ overpressure because of the more stable apparent viscosity data.

The activation energy was determined and the temperature influence on the rheological properties of the pulp was estimated using the Frenkel-Eyring equation:

$$
\mu=A \exp \left(\frac{E_{a}}{R T}\right) \cdot \gamma^{-n}
$$

where $A$ is the pre-exponential multiplier (Pa.s), Ea is the activation energy ( $\mathrm{j} \cdot \mathrm{mol}-1), \mathrm{R}$ is the universal gas constant $(8,314 \mathrm{j} \cdot \mathrm{mol}-1 \cdot \mathrm{K}-1), \mathrm{T}$ is the thermodynamic temperature $\left({ }^{\circ} \mathrm{K}\right)$, $\gamma$ is the numerical value of the strain rate; $n$ is the rate of destruction of the structure.

Under experimental conditions, the strain rate $\gamma$ is a constant value, practically independent of temperature, so the Arrhenius equation was used to determine the energy:

$$
\mu=A \exp \left(\frac{E_{a}}{R T}\right)
$$


Logarithm of the equation (9) received:

$$
\operatorname{Ln}(\mu)=\operatorname{Ln}\left(\mu_{\infty}\right)+\frac{E_{a}}{R} \frac{1}{T}
$$

By entering the notation: $y=\operatorname{Ln}(\mu) ; a=\operatorname{Ln}(\mu \infty) ; b=E a / R ; x=1 / T$, we obtained the linearized equation $y=a+b x$. We determined the coefficients $a$ and $b$, and calculated the pre-exponential multiplier AandEa activation energy. To predict the maximum shear stress of the mezga an attempt was made to determine the type of functional curve from the temperature by reducing the latterto the linear view. The problem was solved by selecting a semi-logarithmic scale (Fig. 7). After analyzing the obtained graphical dependencies in semi-logarithmic scales, we can conclude that equation (9) is applicable for this dependence. The tangent of the angle of inclination of this line determines the activation energy of the process.

Based on mathematical processing of experimental dependencies $\operatorname{Ln}(\mu)$ from $1 / T$, the activation energy and the pre-exponential multiplier are determined. The rectilinear form of the dependence of $\operatorname{Ln}(\mu)$ on 1/T indicates the formation of connections of one type of fluctuation grid. The model flow equation in the framework of the Bingham rheological model taking into account the established temperature influence can be represented by the following dependence:

$$
\mu=A \exp \left(\frac{E_{a}}{R T}\right)=-1.98 \cdot \exp \left(\frac{26.2 \cdot k J}{R T}\right)
$$

The activation energy of the sunflower seed cake for the deformation rate of $3.14 \mathrm{~s}-1$ is $21.19 \mathrm{~kJ} / \mathrm{mol}$, the pre-exponential multiplier $\mathrm{A}=-1.98 \mathrm{~Pa}$.

\section{Conclusion}

Taking into account the fact that the effective viscosity of the pulp corresponds to the viscosity of vegetable oil [18], which is part of this viscoplastic material, we have received confirmationBingham's rheology of sunflower pulp. As can be seen from the presented data, the consistency graph is a Bingham viscoplastic liquid.

Larvae mass demonstrates shear-thinning conduct and found to display HarshleyBuckley flow behavior with yield stress decrease with decreasement in oil content. The larvae mass viscoelastic conduct was described as a weakgel.

Experimental studies have shown the presence of structure formation of the pulp entering the oil press. As the shear rate increases, the effective viscosity decreases. The consistency of the pulp corresponds to Bingham's rheology. It is established that the yield strength of sunflower pulp changes linearly depending on the excess pressure, 
and the obtained dependence $T(\gamma, p)$ allows identifying the flow of the plastic layer as an oil filmat the boundary of the piston flow of the sunflower seed cake. The influence of the increase in oil content of the pulp on the flow rheology is characterized by a decrease in the value of the maximum shear stress of the pulp from 28.5 to $27.11 \mathrm{~Pa}$, which reduces the load on the pressing process.

The structural mechanical and energy properties of sunflower pulp depend on a number of factors: the component composition of the pulp and the temperature conditions of processing. The influence of temperature is characterized by a decrease in the plastic viscosity of the pulp from 0.0985 to $0.0917 \mathrm{~Pa} \bullet$ s for samples at an excess pressure of $1.8 \mathrm{kPa}$.

The information obtained is conceivably helpful for future examinations on the properties of food and process design.

\section{Acknowledgments}

Thanks to Dr. Sergiy Smetana from DIL institute and Dr. Oleksii Parnyakov from Elea company for providing opportunity to study larvae tissue microstructure in DIL institute and for project discussion.

The research was carried out using the equipment of the Research Center for Food and Chemical Technologies of KubSTU (CKP_3111) which development is supported by the Ministry of Science and Higher Education of the Russian Federation (Agreement No. 075-15-2021-679)

\section{References}

[1] Shorstkii I, Khudyakov D, Mirshekarloo MS. (2020). Pulsed electric field assisted sunflower oil pilot production: Impact on oil yield, extraction kinetics and chemical parameters. Innovative Food Science \& Emerging Technologies. 2020;60:102309102318.

[2] Alles MC, SmetanaS, Parniakov O et al. (2020). Bio-refinery of insects with pulsed electric field pre-treatment. Innovative Food Science \& Emerging Technologies.2020. 301;102403-102413.

[3] Makkar HPS, Tran G,Heuzé V, Ankers P. State-of-the-art on use of insects as animal feed. Animal Feed Science and Technology. 2014;197:1-33. 
[4] Han Z, Zeng X, Zhang B, YuS. Effects of pulsed electric fields (PEF) treatment on the properties of corn starch. Journal of Food Engineering. 2009;93(3):318-323.

[5] Xiang BY. Effects of pulsed electric fields on structural modification and rheological properties for selected food proteins.Québec; Pearl Valley Farms LLC. 2008.

[6] Pereira RN, Galindo FG, Vicente AA, Dejmek P. Effects of Pulsed Electric Field on the Viscoelastic Properties of Potato Tissue. Food Biophysics. 2009;4(3):229-239.

[7] Antunes SA, Lanza M, Hense H. Rheological properties of rice bran (Oryza sativa L.) oils processing and soapstock distillation residue. Industrial Crops and Products. 2013;46:111-116.

[8] Gamon G, Evon P, Rigal L. Twin-screw extrusion impact on natural fibre morphology and material properties in poly (lactic acid) based biocomposites. Industrial Crops and Products. 2013;46:173-185.

[9] Muresan V, Blecker C, Danthine S, Racolta E, Muste S. (2013). Confectionery products (halva type) obtained from sunflower: Production technology and quality alterations. A review. Biotechnology, Agronomy and Society and Environment. 2013;17(4):651659.

[10] Akbulut M, Saricoban C, Ozcan MM. Determination of rheological behavior, emulsion stability, color, and sensory of sesame pastes (tahin) blended with pine honey. Food and Bioprocess Technology. 2012;5(5):1832-1839.

[11] Shorstkii I, Khudyakov D. (2020). Influence of pulsed electrical discharge, hydrostatic pressure and temperature on rheological properties of sunflower cake during oil pressing. Heliyon. 2020;6(1):e03046-e03046.

[12] Shorstkii IA, Zherlicin AG, Li P. Impact of pulsed electric field and pulsed microwave treatment on morphological and structural characteristics of sunflower seed. Oilseeds and fats, Crops and Lipids. 2019;26:47.

[13] Akbulut M, Saricoban C, Ozcan MM. Determination of rheological behavior, emulsion stability, color, and sensory of sesame pastes (Tahin) blended with pine honey. Food and Bioprocess Technology. 2012;5(5):1832-1839.

[14] Salehi F, Kashaninejad M. Effect of drying methods on rheological and textural properties, and color changes of wild sage seed gum. Journal of Food Science and Technology. 2015;52(11):7361-7368.

[15] Gukasyan AV, Kosachev VS, Koshevoy YP. Modeling viscous-plastic extrusion of oilbearing materials pertaining to Bingham rheology. IOP Conference Series: Materials Science and Engineering.2019;560(1):012019-012019 
[16] Meher J, Keshav A, Mazumdar B. (2019). Density, steady and dynamic state shear rheological properties of gongura (Hibiscus sabdariffa) leave puree as a function of temperature \& TSS. Carpathian Journal of Food Science \& Technology. 2019;11(4). 81-95.

[17] Ilo S, Schoenlechner R, Berghofe E. Role of lipids in the extrusion cooking processes. Grasas y Aceites.2000;51(1-2):97-110.

[18] Stanciu I. Study concerning the rheological behavior of sunflower oil. Journal of Science and Arts. 2013;4(25):355-360. 